\title{
Translational Medicine - A Multidisciplinary, Collaborative and Global Effort
}

\author{
Wei Lu PhD ${ }^{1}$, Wuwei Feng MD MS ${ }^{2}{ }^{3}$ \\ ${ }^{1}$ School of Life Sciences, Southeast University, Nanjing, China
}

\begin{abstract}
${ }^{2}$ Department of Neurology and Neurosurgery, College of Medicine, Medical University of South Carolina, Charleston, South Carolina
\end{abstract}

\section{${ }^{3}$ Department of Health Science \& Research, College of Health Professions, Medical University of South Carolina, Charleston, South Carolina}

It cost investors $100+$ million dollars and scientists almost 10 years to investigate the clinical effect of the Infrared Laser Therapy for ischemic stroke. But the phase-III clinical trial[1] concluded against the promising results from the phase I and phase II trials[2,3], and it brutally claimed futility that laser therapy has no effect of improving outcomes for patients with acute ischemic stroke. Due to rapid funding withdrawal from the investors, some patient data and protected health information were jeopardized[4].

During the device development process, over 1000 stroke patients at over 100 sites in a dozen countries (including 122 patients in phase I trial[2], 660 patients in phase II trial[3], and 1000 patients in phase III trial supposedly, although only 566 patients completed[1]) were enrolled as a global attempt to test the efficacy of the laser therapy in stroke patients. However, only a few preclinical animal studies investigated this laser technology before moving to stroke subjects. All this was done without knowing the exact mechanism of laser therapy in stroke recovery[5].

What a sad story of translational medicine! So where did the chain break? At the moment, it appears that stroke field is a particularly failure for drug development, with over 2000 neuro-protective molecules that were promising in animal research at one time but all having failed in human research. As a result, Stroke Treatment Academic Industry Roundtable (STAIR)[6] was formed to discuss and address many issues existed in stroke research. Just naming a few: what is the pathophysiology of stroke? What is the natural trajectory of stroke recovery? Why does not the animal model match with the human stroke model? Why do research scientist used young stroke model while stroke generally occurs in elderly humans? Why clinical trials are so expensive but recruitments are so slow?

With numerous lessons of the past, we have to learn and brainstorm new perspective about how we advance translational medicine in a systematic way. It begins with defin- ing what translational medicine is[7]. Some researchers define it as the research process from bench-to-bedside in the development of new drugs, devices, and procedures for patients, while others view it as translating research into clinical practice. While it may mean different things to different people, we feel most of us are acceptable with a "bench-to-bedside T0-T4" approach. In this approach, novel ideas originate from animal research and are tested in animals first (T0), then it moves from animal to human stages in a Phase I trial (T1), subsequently, it goes through Phase II and III trials process (T2) if there is a good signal, and it disseminates to the real world practice by health service research (T3) accordingly, finally it will be assessed to see if it actually improves patients outcomes by population level outcomes research (T4).

Dr. Renyu Liu at the University of Pennsylvania creatively connected a group of research scientists (from T0-T4 stages, from different disciplines, and from different countries) together using social media (Group Wechat, http:// www.wechat.com/en/) for the Journal of the "Translational Perioperative and Pain Medicine (TPPM)"'[8]. It resulted in a heated discussion about perspectives of translational medicine among about 180 participants under the group name of TPPM[9]. Several great ideas were exchanged and proposed, and it signified a unique and important step for a multi-disciplinary, collaborative and global, "benchto-bedside" approach for translational medicine.

Today, China, while a developing country, is an active incubator for biomedical research with clustered worldclass, dedicated, and striving investigators. Many T0 stage research can be easily initiated in China with collaborative efforts from US counterparts to replicate the results. When ready, it can be tested in proof-of-concept study, subsequently in phase II and III in the US where there is a mature system for clinical research. Furthermore, if China could quickly adopt the Good Clinical Practice for clinical 
research, phase II and III trial could be launched in China taking advantage of large patient populations. The positive results from clinical trial could be quickly implemented in clinical practices all over the world thanks to global marketing efforts to improve patient cares. However, such an international collaborative effort could be challenging with issues related to culture differences, language barriers, intellectual property protection, legal issues with funds flow, government regulations etc. Input and support from governments, funding agencies and industries are needed as well.

We hope, in the future, translational medicine will not be lost in "translation" with multidisciplinary, collaborative and global effort.

Correspondence:Wuwei Feng, MD, MS, Department of Neurology \& Neurosurgery, MUSC Stroke Center, Medical University of South Carolina, Tel: 843-792-3020, Fax: 843-7922484, Email: feng@musc.edu

\section{Editor}

Renyu Liu, MD, PhD, Director of Preoperative Medicine, Department of Anesthesiology and Critical Care, Perelman School of Medicine at the University of Pennsylvania

Email: liu@uphs.upenn.edu

Disclosure of Funding

Dr. Feng acknowledges funding from American Heart Association (14SDG1829003), NIH (P20GM109040) and the South Carolina Clinical \& Translational Research Institute/Medical University of South Carolina, through NIH Grant UL1 RR029882 and UL1 TR000062

\section{Additional publication details}

Journal short name: Transl Perioper \& Pain Med

Received Date: March 4, 2015

Accepted Date: March 6, 2015

Published Date: March 10, 2015

Transl Perioper \& Pain Med 2015, 2(1):10-11

\section{Citation and Copyright}

Citation: Lu W, Feng W. Translational Medicine - A Multidisciplinary, Collaborative and Global Effort. Transl Perioper \& Pain Med 2015, 2(1): 10-11

Copyright: (C) $2015 \mathrm{Lu} \mathrm{W.} \mathrm{MD,} \mathrm{MD.} \mathrm{This} \mathrm{is} \mathrm{an} \mathrm{open-ac-}$ cess article distributed under the terms of the Creative Commons Attribution License, which permits unrestricted use, distribution, and reproduction in any medium, provided the original author and source are credited.

\section{References:}

1. Hacke W, Schellinger PD, Albers GW, Bornstein NM, Dahlof BL, et al. (2014) Transcranial laser therapy in acute stroke treatment: results of neurothera effectiveness and safety trial 3, a phase III clinical end point device trial. Stroke 45: 3187-3193.

2. Lampl Y, Zivin JA, Fisher M, Lew R, Welin L, et al. (2007) Infrared laser therapy for ischemic stroke: a new treatment strategy: results of the NeuroThera Effectiveness and Safety Trial-1 (NEST-1). Stroke 38: 1843-1849.

3. Zivin JA, Albers GW, Bornstein N, Chippendale T, Dahlof B, et al. (2009) Effectiveness and safety of transcranial laser therapy for acute ischemic stroke. Stroke 40: 1359-1364.

4. Levine SR, Hill MD (2014) NeuroThera Effectiveness and Safety Trial 3: how do we align corporate and scientific integrity to complete and report pharma-sponsored trials properly? Stroke 45: 3175-3177.

5. Lampl Y (2007) Laser treatment for stroke. Expert Rev Neurother 7: 961-965.

6. Stroke Therapy Academic Industry R (1999) Recommendations for standards regarding preclinical neuroprotective and restorative drug development. Stroke 30: 2752-2758.

7. Rubio DM, Schoenbaum EE, Lee LS, Schteingart DE, Marantz PR, et al. (2010) Defining translational research: implications for training. Acad Med 85: 470-475.

8. Liu R (2014) The launch of the Translational Perioperative and Pain Medicine. Transl Perioper Pain Med 1: 1-2.

9. Hu L, Zhang J, Tu W, Liu R, Zhang L (2015) Perspectives of Translational Medicine. Transl Periop \& Pain Med 2: 12-18. 